Cinémas

Revue d'études cinématographiques

Journal of Film Studies

\title{
Pour poser les jalons d'une narratologie comparée : Léolo à la " recherche d'un temps à jamais perdu »
}

\section{Glenda Wagner}

Volume 9, numéro 2-3, printemps 1999

Les Scénarios fictifs

URI : https://id.erudit.org/iderudit/024793ar

DOI : https://doi.org/10.7202/024793ar

Aller au sommaire du numéro

Éditeur(s)

Cinémas

ISSN

1181-6945 (imprimé)

1705-6500 (numérique)

Découvrir la revue

Citer cet article

Wagner, G. (1999). Pour poser les jalons d'une narratologie comparée : Léolo à la « recherche d’un temps à jamais perdu ». Cinémas, 9(2-3), 175-202.

https://doi.org/10.7202/024793ar
Résumé de l'article

L'auteure est d'avis que lorsqu'on aborde en détail les théories de la narration actuelles (littéraires et filmiques), elles suscitent des embarras à plusieurs égards. C'est en ce sens qu'en s'appuyant sur un film québécois tel Léolo de Jean-Claude Lauzon, dont une parenté certaine de formes lui est apparue avec À la recherche du temps perdu, elle a revisité quelques concepts fondamentaux. Ainsi en est-il de la définition de Genette du mot « récit ». Elle esquisse aussi les prémisses d'une étude comparée de la narration. 


\title{
Pour poser les jalons d'une narratologie comparée: Léolo à la "recherche d'un temps à jamais perdu " ${ }^{1}$
}

\section{Glenda Wagner}

\begin{abstract}
RÉSUMÉ
L'auteure est d'avis que lorsqu'on aborde en détail les théories de la narration actuelles (littéraires et filmiques), elles suscitent des embarras à plusieurs égards. C'est en ce sens qu'en s'appuyant sur un film québécois tel Léolo de Jean-Claude Lauzon, dont une parenté certaine de formes lui est apparue avec $A$ la recherche $d u$ temps pertu, elle a revisité quelques concepts fondamentaux. Ainsi en est-il de la définition de Genette du mot "récit". Elle esquisse aussi les prémisses d'une étude comparée de la narration.
\end{abstract}

\section{ABSTRACT}

This author takes the position that in treating in detail current theories of narration, both literary and cinematic, confusion arises in several respects. It is from this perspective that she uses the Quebec film Léolo by Jean-Claude Lauzon (in which she sees common formal features with $\dot{A}$ la recherche $d u$ temps perdu) to revisit some fundamental concepts. Genette's definition of narrative (récit) figures prominently, in addition to the sketching out of a comparative study of narration.

La critique a été très partagée à propos de Léolo ${ }^{2}$ de JeanClaude Lauzon (1992), mais on s'est entendu pour dire que le film contenait trop de fragments de souvenirs en désordre. Tel 
n'est pas mon avis, et je vais tenter de démontrer, à l'aide de la narratologie, que la structure temporelle de Léolo ressemble, à plus d'un titre, à celle d'À la recherche du temps perdu de Marcel Proust. En effet, dans la construction du récit, Lauzon fait grand usage et de la scène et de l'itératif. Pour faire le parallèle entre Léolo et la Recherche de Proust, je traiterai de questions de vitesses, de fréquence et d'ordre narratifs relevant du Temps narratif.

Bien que les trois classes fondamentales de la narratologie (Voix, Mode et Temps) soient à ce point intrinsèquement liées ${ }^{3}$ qu'on ne puisse parler de l'une sans évoquer l'autre, leur pertinence relative dans l'analyse d'une ouvre peut varier beaucoup. En ce qui regarde Léolo, s'il est indéniable que ce film exige une étude systématique du Temps narratif - les prouesses temporelles étant au cœur même de l'originalité de l'œuvre -, le choix de l'auteur de faire raconter "toute" l'histoire par l'un de ses "personnages", lequel tient le rôle éponyme du film, s'avère également intéressant d'un point de vue théorique. Dans mon étude, la pratique agira donc sur la théorie, et vice versa. Après avoir d'abord identifié cette voix et du même coup dévoilé un hiatus dans le vocabulaire narratologique filmique courant, je me pencherai brièvement sur le parti pris modal du récit, avant de définir la spécificité temporelle de Léolo.

\section{Qui raconte dans Léolo?}

Dès l'incipit du film, on entend une voix off qui dit: "Ça, c'est chez "moi" dans le quartier du Mile-End, à Montréal, au Canada." Cette voix, qui est celle d'un narrateur s'adressant d'emblée au spectateur virtuel, parlera tout au long du film. Comme pour la Recherche de Proust, nous avons affaire à la présence massive d'un narrateur explicite. Celui-ci n'apparaît jamais dans le récit de son enfance, qui se termine à l'âge de 12 ans. La narration est ultérieure. Et jamais l'histoire ne vient rejoindre la narration, la vie de Léo s'étant cristallisée à sa douzième année.

Comment expliquer que les théoriciens du cinéma sont toujours paralysés par le fait que le narrateur verbal ne peut être tenu, entre autres choses, responsable des images mouvantes, alors que les littéraires restent indifférents au fait que des récits 


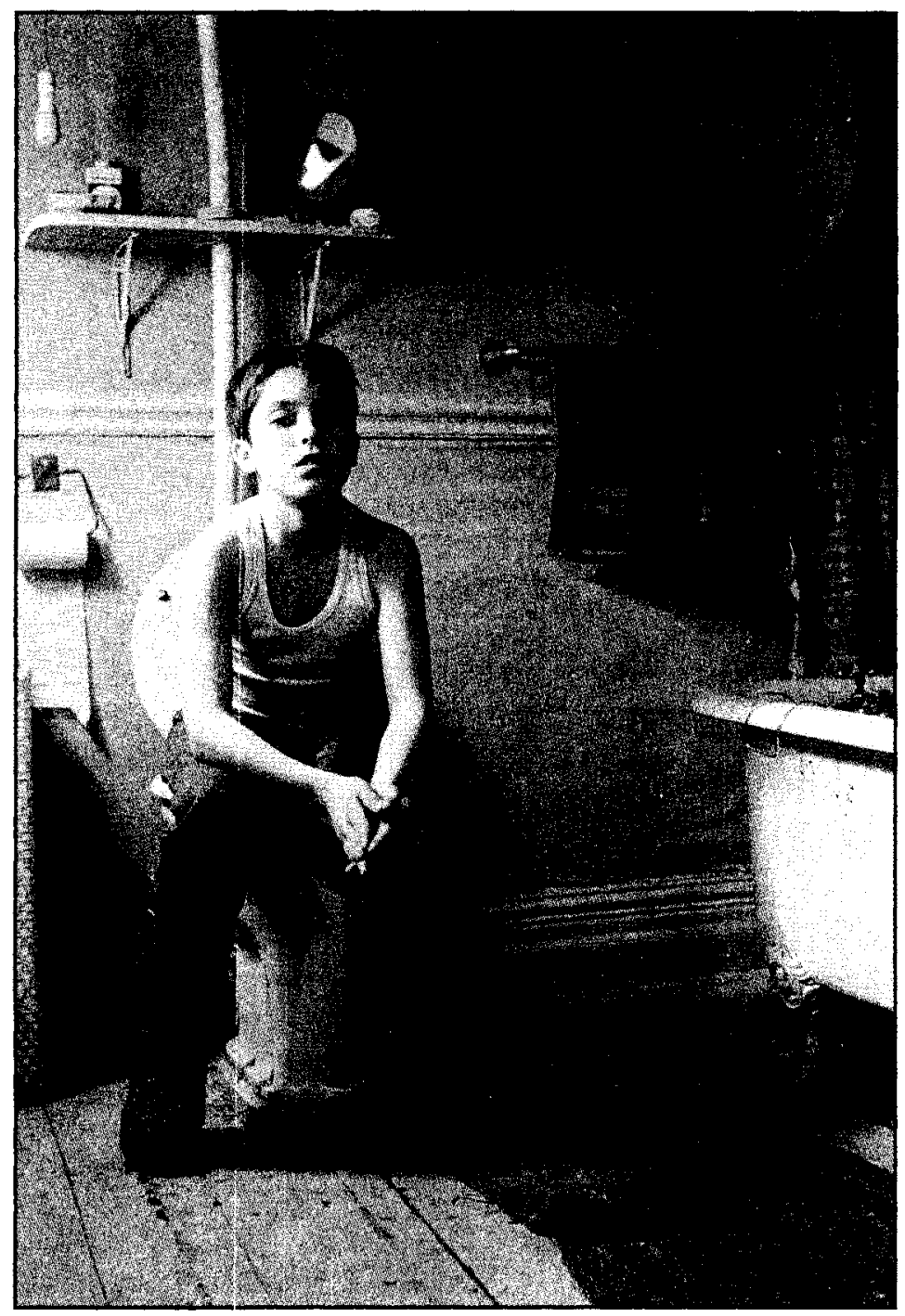

Léolo de Jean-Claude Lauzon (1992)

tels que la Recherche sont présentés sous forme écrite, sans que le narrateur, Marcel, n'ait écrit un seul mot? Genette, qui relève ce phénomène, notamment en ce qui concerne Les Lauriers sont coupés, conclut qu'il est "[...] mystérieusement capté et transcrit par Dujardin" (1972, p. 240). Et Metz d'ajouter - ce qui, en général, est admis:

Pour poser les jalons d'une narratologie comparée: Léolo à la «recherché d'un temps..." 
[Le] texte écrit donne toujours, à des degrés divers, l'impression d'une présence énonciative: [...] "Si ça parle, c'est que quelqu'un parle": tel est le sentiment général, même s'il s'agit d'un livre. Mais l'équivalent filmique de cette certitude intérieure et immédiate est loin d'être assuré. "S'il y a des images à voir, c'est que quelqu'un les a arrangées": voilà qui ne recueille pas l'adhésion des foules (Metz, 1987, p. 20 et 1991, p. 18).

La différence entre livre et images, est-ce un élément à prendre en compte en narratologie?

\section{Voix narrative: la métaphore}

Comme on sait, Gérard Genette a utilisé, dans un sens métaphorique, le concept de Voix (emprunté à la grammaire du verbe) pour parler de cette instance narrative à laquelle il incombe de prendre la parole pour raconter son récit. Cette instance narrative est non seulement ce sujet qui accomplit ou subit l'action, mais surtout celui qui la rapporte. Pour l'identifier dans le texte romanesque, deux types de narration de base ont été répertoriés, sur lesquels se greffe un troisième: hétérodiégétique (narrateur absent de l'histoire, ou étranger ${ }^{4}$ ), homodiégétique (narrateur présent comme personnage) et autodiégétique (degré fort de l'homodiégéticité); de plus, divers niveaux narratifs ont été distingués: extradiégétique (instance narrative du récit primaire), intradiégétique (instance narrative du récit métadiégétique), etc. Faisant partie intégrante de la situation narrative, la position du narrataire est symétrique de celle du narrateur. Pour ce qui est du texte filmique, Gaudreault propose les termes de narrator (équivalent du narrateur extradiégétiquehétérodiégétique de Genette) et de narrateur délégué (narrateur intradiégétique-homodiégétique de Genette); Jost, quant à lui, oppose le narrateur implicite (équivalent du narrator de Gaudreault) au narrateur explicite (narrateur délégué de Gaudreault). Pour sa part, Metz n'utilise, en fin de compte, qu'un seul terme pour la production et un seul pour la réception: énonciation et spectateur; tandis que Casetti ${ }^{5}$ scinde l'énonciation en deux, c'est-à-dire en énonciateur et en énonciataire, etc. Metz entend par énonciation «[...] l'acte sémio- 
logique par lequel certaines parties d'un texte nous parlent de ce texte comme d'un acte» (1987, p. 22), alors que chez Casetti, "[...] l'énonciation, et avec elle ce que nous pourrions désigner comme son sujet, n'apparaissent [sic] jamais en tant que tels. L'énonciation ne se donne que dans l'énoncé dont elle est le présupposé" (p. 42). Pour Casetti, trois instances sont toujours présentes, même si c'est de façon variable, dans le texte filmique: le Je (qui représente l'énonciateur filmique), le Tu (qui désigne l'énonciataire, l'instance à qui s'adresse l'énoncé) et, enfin, le Il (qui renvoie à l'énoncé lui-même). D'entrée de jeu, au chapitre sur Voix de Figures III, Genette met en parallèle énonciation et narration:

On sait que la linguistique a mis quelque temps à entreprendre de rendre compte de ce que Benveniste a nommé la subjectivité dans le langage, c'est-à-dire de passer de l'analyse des énoncés à celle des rapports entre ces énoncés et leur instance productrice - ce que l'on nomme aujourd'hui leur énonciation. Il semble que la poétique éprouve une difficulté comparable à aborder l'instance productrice du discours narratif, instance à laquelle nous avons réservé le terme, parallèle, de narration (1972, p. $\left.226^{\circ}\right)$.

Benveniste, de surcroît, nous incitait à prendre " $[\ldots]$ garde à la condition spécifique de l'énonciation: c'est l'acte même de produire un énoncé et non le texte de l'énoncé qui est notre objet" (p. 80).

$\mathrm{Si}$, quant au narrateur, la terminologie genettienne fait l'unanimité parmi les narratologues du littéraire (Bal, Vitoux, Cohn, Lintvelt, etc.), ceux du filmique, par contre, n'ont pas trouvé de consensus. À l'instar de Cohn, on sait que

[...] la valeur heuristique d'un terme critique bien défini consiste précisément à permettre d'identifier chaque occurrence individuelle comme l'exemple d'une norme générale et une variation par rapport à cette norme [et qu'une] appellation commune pour un phénomène $[\ldots]$ répandu [a] au moins l'intérêt de simplifier le discours critique (p. 132). 


\section{Statuts des narrateurs : la répétition}

En narratologie littéraire, il y a quatre paradigmes possibles: narrateur extradiégétique-hétérodiégétique (de L'Odyssée ou de L'Iliade) ou homodiégétique (Marcel, de la Recherche), et narrateur intradiégétique-hétérodiégétique (Schéhérazade, des Mille et Une Nuits) ou homodiégétique (Des Grieux, de Manon Lescaut). On ne les trouve pas dans la narratologie filmique la plus courante. Gaudreault et Jost en proposent deux. Le narrator de Gaudreault est, en effet, l'équivalent du narrateur extradiégétiquehétérodiégétique littéraire - jamais du narrateur extradiégétiquehomodiégétique, parce qu'on le dit impossible à incarner au cinéma — tandis que le narrateur délégué ${ }^{7}$, narrateur-personnage de l'histoire, est toujours une instance enchâssée. Aussi ne peut-il être qu'intradiégétique et n'est tenu responsable que de sa voix, car les autres matières de l'expression filmique relèvent du narrator ${ }^{8}$. On l'a vu, Jost, avec d'autres mots, circonscrit des instances identiques à celles de Gaudreault. Qui plus est, le narrateur implicite de Jost "[...] ne fait rien à proprement parler, il ne réalise rien $[\ldots]$, c'est une idée transcendantale qui a un usage immanent, la lecture du film [...]. Les choses du récit doivent être considérées comme si elles tenaient leur existence d'une instance supérieure» (1990, p. 138). À propos des enchâssements, Bordwell avait déjà suggéré: "On the principle that we ought not to proliferate theoretical entities without need, there is no point in positing communication as the fundamental process of all narration, only to grant that most films "efface" or "conceal" this process" (p. 62). Quant aux termes de Metz et de Casetti, ce sont des génériques, il n'y a plus de paradigme.

Non plus que de métaphore possible...

Ne se situant pas au niveau terminologique, l'accord entre les narratologues du filmique, somme toute, se retrouve ailleurs... Il est dans leur façon d'appréhender leur objet: le film. Tous (sauf Branigan), semble-t-il, cherchent à justifier l'une ou l'autre des cinq matières de l'expression filmique circonscrites par Metz: les images mouvantes, les bruits, les mentions écrites, le son, la musique. C'est pourquoi la narration n'émerge jamais autrement que comme une figure "enchâssée", qui n'a pas d'autonomie". "Depuis quelques années, le débat est largement terminologique. 
Chacun propose cles termes [...] dans l'espoir de faire avancer les idées et de mieux parler de l'objet qui nous préoccupe: le film, le récit cinématographique, etc. Ces débats se font dans l'illusion d'un consensus [...]" (Jost, 1990, p. 138). Sans les nombreux avatars qu'a connus l'objet de la narratologie, la désillusion de Jost n'aurait peut-être pas été: d'un récit singulier, on est passé au récit en général, et du récit filmique au film, c'est-à-dire aux procédés propres du langage cinématographique, etc.

Proposer une narratologie comparée largement inspirée de la théorie de Genette est périlleux. D'autant que l'on connaît les réserves de Genette à l'égard du statut du film comme récit ${ }^{10}$. Pour ce dernier, le texte filmique n'est pas à proprement parler un récit, parce qu'il n'est pas un être de langue ". Aussi, non seulement Genette distingue-t-il le récit «au sens étroit» (auquel appartient le récit littéraire) du récit "au sens large » (qui admet le récit filmique), mais encore il désigne comme l'objet d'étude exclusif de la narratologie le récit "au sens étroit». Or, Genette est pour le moins ambigu (ou louvoyant) en ce qui concerne l'objet de "sa" narratologie. Comme le précise Benveniste: "[...] on ne concevrait pas une science ${ }^{12}$ incertaine de son objet, indécise sur son appartenance» (p. 47).

\section{L'objet de la narratologie: le paradoxe}

Il y a en effet des discordances entre Figures III et Nouveau Discours du récit quant à l'objet de la narratologie. Dans Figures $I I I$, Genette joue sur deux tableaux à la fois; son premier objet, $\grave{A}$ la recherche $d u$ temps perdu, appartient à la critique et à l'axe diachronique, tandis que, son second objet, le récit en général, correspond à la théorie et à l'axe synchronique. Dans Nouveau Discours du récit, par contre, Genette n'évoque plus le récit de Proust comme objet de la narratologie. Ce silence s'explique peut-être par le fait que Genette aurait pris conscience que sa narratologie pouvait devenir une science, alors qu'il est connu, au moins depuis Saussure, qu' "il n'y a de science que du général ». Dans Nouveau Discours du récit, Genette affirme au contraire que la narratologie porte sur le récit et la narration, et non sur l'histoire. C'est de cette triade que sera dégagé l'objet de la narratologie. 
Genette a redéfini les trois termes récit / narration / histoire à partir de la définition ambiguë qui est donnée au mot récit. Celui-ci a ailleurs qu'en narratologie au moins trois acceptions. Un premier sens, que Genette conserve, veut qu'il désigne l'énoncé narratif, le discours oral ou écrit qui assume la relation d'un ou de plusieurs événements. Un deuxième sens, que Genette attribue à l'histoire, veut que le récit désigne une succession d'événements réels ou fictifs, qui font l'objet du discours. Un dernier, qui revient à la narration, est que le récit désigne un événement qui consiste en ce que quelqu'un raconte quelque chose: l'acte de narrer pris en lui-même. Genette propose:

[...] sans insister sur les raisons d'ailleurs évidentes $d u$ choix des termes, de nommer histoire le signifié ou contenu narratif $[\ldots]$, récit proprement dit le signifiant, énoncé, discours ou texte narratif lui-même, et narration l'acte narratif producteur et, par extension, l'ensemble de la situation réelle ou fictive dans laquelle il prend place (1972, p. 72).

Faut-il avoir oublié que le signifiant et le signifié sont insécables?

L'entité linguistique n'existe que par l'association du signifiant et du signifié; dès qu'on ne retient qu'un de ces éléments, elle s'évanouit ; au lieu d'un objet concret, on n'a plus devant soi qu'une pure abstraction (Saussure, p. 144).

[La] langue est [...] comparable à une feuille de papier : la pensée est le recto et le son le verso; on ne peut découper le recto sans découper en même temps le verso; de même dans la langue on ne saurait isoler ni le son de la pensée, ni la pensée du son; on n'y arriverait que par abstraction dont le résultat serait de faire de la psychologie pure ou de la phonologie pure (p. 157).

Chez Genette, donc, le signifiant et le signifié sont distribués comme suit :

$$
\begin{aligned}
& \text { Récit = signifiant } \\
& \text { Histoire = signifié }
\end{aligned}
$$


Voici, sous forme schématique, une transcription des termes genettiens :

récit $=$ narration + histoire
texte narratif $=$ acte narratif + contenu narratif
signifiant $=\ldots \ldots \ldots$ signifié

Pour que l'équation recouvre son équilibre, il faut que le signifiant retrouve sa place auprès du signifié, ce qui ne peut se réaliser que d'une manière:

$$
\begin{gathered}
\text { récit }=\text { narration }+ \text { histoire } \\
\text { texte narratif }=\text { acte narratif }+ \text { contenu narratif } \\
\text { signe }=\text { signifiant }+ \text { signifié }
\end{gathered}
$$

La narratologie genettienne est formelle. "Discours du récit" "[...] porte sur le récit et la narration, non sur l'histoire" (Genette, 1983, p. 106), c'est-à-dire le contenu.

Lui reprocher de [le] négliger, c'est lui reprocher son choix d'objet. Je conçois d'ailleurs assez bien une telle critique: pourquoi me parlez-vous des formes, alors que seul le contenu m'intéresse? Mais si la question est légitime, la réponse est trop évidente: chacun s'occupe de ce qui le point, et si les formalistes n'éraient pas là pour étudier les formes, qui voudrait s'en charger à leur place (p. 107)?

Pour cerner l'objet de la narratologie, il reste à isoler un terme:

- Histoire + récit $=$ narration
ou encore
Narration $=$ récit - histoire
Signifiant $=$ signe - signifié
équation qui découle de
Récit $=$ narration + histoire
Récit $=$ signifiant + signifié

Pour poser les jalons d'une narratologie comparée: Léolo à la "recherche d'un temps..." 
Il s'ensuit donc que le récit ne peut pas être l'objet de la narratologie. Il faut lui substituer la narration. En d'autres mots, les théoriciens du filmique ne travaillent pas à partir du bon objet. Leur préoccupation ne devrait pas être le texte filmique (le Il de Casetti), mais la narration qui l'a mis au jour (le Je de Casetti), ce qui fait sens, car on sait bien que "[...] l'ordre véritable [est] l'acte narratif instaurant (inventant) à la fois l'histoire et son récit" (Genette, 1983, p. 11). Quant aux théoriciens du littéraire, qui restent indifférents au fait que le texte (le récit) ait été écrit ou non par ledit narrateur, ils semblent faire fi de leur objet. Ou, contre vents et marées, et pour paraphraser Benveniste, auront-ils compris que "c'est l'acte même de produire un énoncé et non le texte de l'énoncé qui est [leur] objet».

\section{Ressemblance des narrateurs littéraires et filmiques: l'autre métaphore}

Le temps est venu de combler la lacune dans la typologie filmique en ce qui regarde le narrateur extradiégétique-homodiégétique. Pour ce faire seront examinées, dans un premier temps, la notion de "personne" et, dans un second temps, celle des niveaux. On verra que la notion de "personne" a été, à juste titre, critiquée; et que n'eût été de l'objet de la narratologie genettienne, le récit, il n'aurait pas été nécessaire de toujours recourir à une instance extradiégétique-hétérodiégétique filmique. Comme l'écrit Olivier Reboul: "Pour comprendre une métaphore, il faut savoir saisir la ressemblance»; elle est «[...] un sentiment global». Le pouvoir de la métaphore? "[Pouvoir] de nommer, de créer par ressemblance une vraisemblance qui n'existerait pas sans elle» (p. 46).

Pour revenir à la narratologie, je souligne que la notion de "personne", telle qu'elle a été développée par Genette, n’a pas reçu l'adhésion de tous les théoriciens du littéraire. Pour s'en convaincre, il suffit de comparer les passages suivants, où, tour à tour, Genette, Bal et Hamburger prennent position:

Je veux réitérer mes réserves à l'égard du terme même de personne, que je ne maintiens que par concession à l'usage, en rappelant qu'à mes yeux tout récit est, explicitement ou non, "à la première personne", puisque 
son narrateur peut à tout moment se désigner luimême par ledit pronom. [...] La distinction courante entre récits "à la première " et "à la troisième" personne opère donc à l'intérieur de ce caractère inévitablement personnel de tout discours (Genette, 1983, p. 65).

Quant à Bal, dans Narratologie. Les instances du récit, elle écrit:

Il n'existe $[\ldots]$ qu'une seule "personne" narrative: la première personne. Le narrateur [...] peut être "visible", présent dans le récit, ou absent. [...] [Le] narrateur, pour être présent, doit l'être «à l'intérieur" du récit. C'est donc le narrateur homodiégétique de Genette $[\ldots]$. Il peut aussi être un narrateur hétérodiégétique, qui raconte "à la première personne» une histoire [de laquelle] il est absent. Le narrateur «à la troisième personne" n'existe pas (p. 34).

Hamburger, pour sa part, s'exprime, dans Logique des genres littéraires, comme suit:

Certes, il est commode, lorsqu'on décrit une fiction "narrative", d'uriliser cetre expression personnifiante; il est vrai que la narration est parmi les moyens d'expression artistiques celui qui peut le plus donner l'impression d'instaurer une relation entre une "personne" et les personnages qu'il crée, ainsi du reste qu'avec le lecteur. On n'échappe pas non plus à la personnification du narrateur quand on parle de "narrateur fictif ", qui ne s'identifie pas, au niveau biographique, avec l'auteur réel. Il n'existe pas de narrateur fictif qui serait une projection de l'auteur, "une figure créée par l'auteur", pas même où l'auteur désigne le Je par des figures rhítoriques telles que "notre héros" [...]. Il n'y a que l'auteur et ses narrations. On ne peut parler d'un narrateur fictif que dans le cas où l'écrivain "crée" ce narrateur, ce qui correspond au narrateur à la $1^{\text {rc }}$ personne dans les cas concernés. [...] Traditionnellement, lorsqu'on pense à un narrateur, on pense justement au narrateur à la 3 c personne (p. 128).

Quoique tout le monde s'accorde pour reconnaître le narrateur "à la première personne", il n'en va pas de même pour 
l'autre narrateur, celui que Genette nomme précisément hétérodiégétique. En bref, il est difficile de suivre Genette dans la concession qu'il fait à l'usage, puisqu'il s'agit d'un paradoxe. Bal et Hamburger ont toutes les deux raison. Le narrateur, c'est celui qui parle, alors que la troisième personne grammaticale est celle qui ne parle pas et à qui on ne parle pas, mais dont on parle. Bal n'a offert aucune solution; Hamburger propose la notion de fonction narrative en remplacement du narrateur. Cette proposition, si séduisante soit-elle, rencontre un obstacle: l'usage. Comme Cohn l'a remarqué, on n'a d'autre choix que de parler de narrateur - même dans le cas de Hamburger - tant l'habitude est prise. Pour échapper à cette aporie et pouvoir continuer d'utiliser la notion de narrateur, force est d'aller chercher celui qui parle. Et c'est l'auteur, qui ne délègue sa narration à aucune "personne». Genette n'a-t-il pas écrit: «[...] le narrateur extradiégétique se confond totalement avec l'auteur - je ne dirai pas, comme on le fait trop, "implicite", mais bel et bien explicite et proclamé» (1983, p. 92) ? S'impose alors à nous le questionnement suivant: pourquoi reconnaissait-on à Schéhérazade, dans Les Mille et Une Nuits, le statut de narratrice intradiégétique-hétérodiégétique si l'on refusait à Anne Hébert, dans Kamouraska, celui de narratrice extradiégétiquehétérodiégétique? Tout comme Schéhérazade, Anne Hébert est étrangère à l'histoire, qui n'est pas la sienne, et n'a pas été témoin des événements rapportés. Tout comme Schéhérazade, elle est auteure de la narration tant qu'elle ne l'a pas déléguée. Par contre, leur niveau de narration n'est pas le même: Schéhérazade est intradiégétique, mais Anne Hébert est extradiégétique. Pourquoi donc ne pas admettre qu'Anne Hébert, dans certains passages du roman (tel celui-ci: "L'été passa en entier. Mme Rolland, contre son habitude, ne quitta [...]»), soit hétérodiégétique (parce qu'elle n'a pas l'occasion d'employer la première personne pour désigner l'un des personnages de l'histoire) et extradiégétique (puisqu'elle est la première à parler, sans déléguer sa narration, ce qu'elle fera par la suite) ? C'est cela même qui l'autorise - discussion qui relève du Mode - à sonder les cœurs et les reins de ses protagonistes, ce qu'aucun personnage ne peut faire sans infraction. Tout cela sapplique autant 
à Jutra, qui a adapté (raconté) l'histoire de Kamouraska à l'écran. Lorsqu'on a évacué l'auteur de la théorie, c'était pour contrer des analyses telles que celles de Sainte-Beuve - sa méthode consistant, on le sait, à ne pas séparer l'homme de l'œuvre. Étant donné nos connaissances actuelles, analystes et théoriciens sauront faire la part des choses. Il y a bien sûr la phrase fameuse de Proust: «[...] qu'un livre est le produit d'un autre moi que celui que nous manifestons dans nos habitudes, dans la société, dans nos vies» (1954, p. 127).

Proust lui-même, écrira Barthes dans son article "La mort de l'auteur ", se donna pour tâche de brouiller inexorablement, par une subtilisation extrême, le rapport de l'écrivain et de ses personnages: en faisant du narrateur non celui qui a vu ou senti ni même celui qui écrit, mais celui qui "va écrire» (le jeune homme du roman - mais, au fait, quel âge a-t-il et qui est-il ? - veut écrire, mais il ne le peut, et le roman finit quand enfin l'écriture devient possible (p. 65).

Genette, dans Palimpsestes, ira jusqu’à poser la question: "Proust structuraliste?", et enchaînera sur "Je n’ai rien dit» (p. 117). Pour ainsi dire naturellement, la question inverse apparaît: les narratologues sont-ils proustiens? Quoi qu'il en soit, la relation homodiégétique de Marcel dans la Recherche peut se formuler ainsi : $A \neq N=P$, alors que la relation hétérodiégétique de Schéhérazade et d'Anne Hébert s'énoncerait: $A=N \neq P$. Dans les deux cas, l'auteur n'est pas le personnage, puisque si $\mathrm{N}=\mathrm{P}$ et $A \neq N$, alors $A \neq P$; et si $N \neq P$ et $A=N$, donc $A \neq P$. Cela devrait suffire à les inscrire dans le régime de la fiction. Comme l'exprime Genette dans Fiction et diction, la dissociation du personnage et du narrateur définit, en fiction et ailleurs, le régime hétérodiégétique, de la même manière que leur identité détermine le régime homodiégétique. La dissociation de l'auteur et du personnage définit le régime de l'allographie, qu'elle soit fictionnelle (hérérodiégétique, comme dans Tom Jones, ou homodiégétique, comme dans Gil Blas) ou factuelle (généralement hétérodiégétique, comme en Histoire ou en biographie), alors que leur identité définit celui de l'autobiographie ${ }^{13}$. Enfin, s'il faut convaincre encore, Genette convient que «[...] dans 
une large mesure le récit de fiction hétérodiégétique est une mimèsis de formes factuelles comme l'Histoire" (1991, p. 90).

De la discussion qui précède me semblent découler trois conséquences sur le plan méthodologique:

- On ne devrait pas multiplier les instances inutilement. $\grave{A}$ ce propos, je cite Genette: "Il y a dans le récit, ou plutôt derrière ou devant lui, quelqu'un qui raconte, c'est le narrateur. Au-delà du narrateur, il y a quelqu'un qui écrit et qui est responsable de tout son en-deçà. Celui-là, grande nouvelle, c'est l'auteur (tout court), et il me semble, disait déjà Platon, que cela suffit" (1983, p. 102).

- On doit continuer d'utiliser, à l'instar de Cohn, le terme "narrateur» et ses dérivés narratologiques.

- Il conviendrait d'inscrire, au moins dans la théorie (et sans guillemets de protestation!), le nom de l'auteur réel ou fictif en régime hétérodiégétique, comme Genette l'a déjà fait dans son tableau de Figures III (p. 256).

La position théorique que je viens de préciser m'amène à penser qu'il serait maintenant opportun de réintroduire l'auteur réel dans la théorie, et de faire une entorse au principe d'immanence de la narratologie; je lui attribue une position identique à celle de l'auteur fictif (tel que défini par Genette) si bien que tous les deux, autant pour le récit littéraire que le récit filmique, assumeraient - sans discrimination - la fonction de narrateur hétérodiégétique. Cela veut dire que ces narrateurs sont étrangers à l'histoire et que le récit en cause n'est pas une autobiographie. Les narrateurs extradiégétiques-hétérodiégétiques du littéraire et du filmique s'avéreraient donc identiques. En ce qui a trait au principe d'immanence, il faut savoir que Genette luimême l'ignore quand celui-ci le dessert. Pour preuve, lorsqu'il applique sa théorie à la Recherche, l'auteur de Figures III justifie la prise en compte des deux ouvres de Proust (la Recherche et Jean Santeuil) en déclarant que "[...] la Recherche, moins qu'aucune [œuvre], ne peut être considérée [comme] une œuvre close» (1972, p. 67). Genette encore, lorsqu'il conclut sur certaines paralepses de Proust, écrit: "Tout cela, et bien d'autres choses, vient de Proust et nous ne pousserons pas le dédain du "référent" jusqu'à feindre de l'ignorer; mais de tout cela, nous le 
savons aussi, il a voulu se décharger en en déchargeant [sic] son héros» (p. 259). La position de la narratologie actuelle est intenable. Dans la citation précédente, il y a à la fois la référence supposée (immanente), le héros, et la référence obligée (transcendante) à l'œuvre romanesque, l'auteur. L'immanence appelle toujours la transcendance, son double obligé. Aussi, bien qu'il soit préférable dans un premier temps de privilégier l'approche immanente pour analyser une œuvre poétique, est-il impossible de faire totalement abstraction d'une approche transcendante dans un dernier temps. De plus, comme George Steiner l'a rappelé dans Réelles présences. Les arts du sens, le sens est vertical, dans l'œuvre et en dehors d'elle. Dès lors, n'est-il pas à propos de s'interroger sur la stricte immanence de la narratologie, sans que cela implique l'abandon de la théorie genettienne?

Pour ce qui est des narrateurs extradiégétiques-homodiégétiques filmiques, la théorie actuelle a conclu à leur improbabilité. On l'a vu, Gaudreault départage la responsabilité du récit filmique. Jost fait de même. Metz, à son tour, affirme que le narrateur en voix off « $[\ldots]$ ne peut jamais faire comme si l'énonciation première, véritable, n'était celle du "Grand Imagier" évoqué par Albert Laffay" (1991, p. 18). Bordwell, enfin, refuse de créer des entités théoriques pour rien, la narration devient le fait d'un percepteur, mais non d'un narrateur (p. 62). Pourtant, chez les littéraires, on accepte que la narration extradiégétique ne soit pas forcément ni écrite ni même parlée; et, inversement, que la narration intradiégétique ne soit pas nécessairement le produit d'une narration orale, qu'elle puisse consister en un texte écrit, ou se donner ouvertement comme un monologue autonome, ou être assumée par une représentation non verbale - le plus souvent visuelle (Genette, 1972, p. 240). Ces dernières considérations sont possibles grâce à la métaphore. Appliquées au filmique, elles permettent d'envisager un autre type de narrateur, celui que l'on nomme extradiégétique-homodiégétique, voire autodiégétique et ce, en ne se basant que sur la narration et non sur celui qui serait responsable de la mise en œuvre (forme) des cinq matières de l'expression filmique. Son rôle n'est pas de crever l'écran, c'est-a-dire " [...] de faire un effet remarquable dans un film (en parlant d'un acteur de cinéma)" (Le Petit Robert). 
Le narrateur est différent du personnage (et, a fortiori, de l'acteur). Dans La Comtesse aux pieds nus (Joseph Mankiewicz, 1954), par exemple, Bogart, qui interprète le rôle d'Harry Dawes, crève l'écran en tant qu'acteur, mais non comme narrateur homodiégétique. En effet, à titre de narrateur, Harry Dawes (Bogart) n'est pas visible, mais dissimulé derrière le personnage qu'il incarne. Il faut se rappeler que "tout événement raconté est à un niveau immédiatement supérieur à l'acte producteur". Dawes (Bogart) est extradiégétique comme narrateur (signifiant) et intradiégétique comme acteur (signifié). En définitive, ce qui permet d'identifier un narrateur extradiégétiquehomodiégétique, comme cela se fait chez les littéraires, c'est qu'il ne s'adresse à aucun personnage de l'histoire, tout au plus au public virtuel.

Qui raconte dans la Recherche? C'est Marcel, narrateur extradiégétique-autodiégétique. Dans Léolo? Une fois de plus, il s'agit d'un narrateur extradiégétique-autodiégétique: Léo ou Léolo. Il n'est aucun besoin ici du Grand Imagier de Laffay, du narrator de Gaudreault, du narrateur implicite de Jost, etc., puisque la justification des cinq matières de l'expression relève d'une autre théorie que de la narratologie.

\section{Qui perçoit dans Léolo?}

Pour l'étude du Mode, il ne sera question que de la notion genettienne de focalisations. Par focalisations, Genette entend une sélection de l'information narrative par rapport à ce que la tradition nommait l'omniscience. "L'instrument de cette (éventuelle) sélection est un foyer situé, c'est-à-dire [...] une sorte de goulot d'information, qui n'en laisse passer que ce qu'autorise sa situation" (Genette, 1983, p. 49). Quand la voix off dit: "Parce qu'il se tenait caché, je n'ai jamais vu le visage de mon vrai père", ce visage est également caché au spectateur. Léolo est en focalisation interne, c'est-à-dire que

[...] le foyer coïncide avec [le] personnage, qui devient alors le "sujet" fictif de toutes les perceptions, y compris celles qui le concernent lui-même comme objet: le récit peut alors nous dire tout ce que le personnage perçoit et tout ce quil pense $[\ldots]$; il ne 


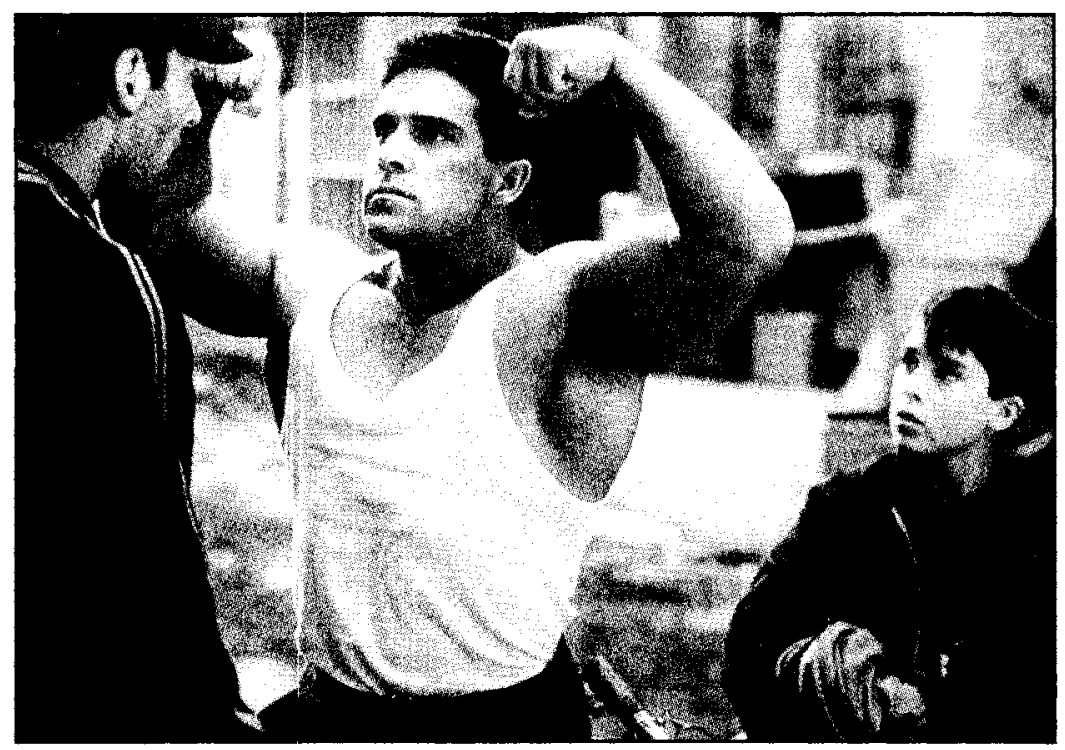

\section{Léolo de Jean-Claude Lauzon (1992)}

"doit" en principe rien dire d'autre; s'il le fait, c'est [...] une altération (paralepse), c'est-à-dire une infraction délibérée ou non au parti modal du moment (Genette, 1983, p. 49).

Il y a certes des paralepses dans Léolo. En effet, on attribue parfois au personnage focal des souvenirs qui ne peuvent lui appartenir en propre, puisqu'il était lui-même vraisemblablement absent lors des événements. C'est le cas lorsqu'on apprend que c'est le poète-dompteur de vers qui avait mis le livre L'Avalée des avalés de Réjean Ducharme sous une patte de la table de la cuisine, ce qui est en contradiction avec les dires de la voix off du début: "C'était le seul livre de la maison. Je ne me suis jamais demandé comment il était arrivé là." Il en va de même lors de la visite de la mère et de Fernand à l'école (scène où les images dépassent la perception de Léo) et dans l'échange de paroles entre un professeur de français et le dompteur de vers sur les écrits du gamin (probablement une vision de Léo). 


\section{Quelles sont les modalités temporelles de Léolo?}

Autrement dit, dans quel ordre et à quelles vitesses et fréquence la narration évolue-t-elle par rapport à l'histoire? Relèvent de l'ordre les phénomènes d'anticipation (analepse) et de rétrospection (prolepse); de la vitesse, la rapidité (ellipse) ou la lenteur (pause descriptive) de la narration, et de la fréquence, l'itératif, le répétitif et le singulatif. À partir de ces trois déterminations genettiennes (ordre, vitesses, fréquence), je serai en mesure d'expliquer les relations qui existent entre le temps de l'histoire (celui des événements) et le temps de la narration ${ }^{14} \mathrm{de}$ Léolo (celui de l'acte narratif).

Étudier l'ordre, c'est confronter l'ordre de disposition des événements dû à la narration à l'ordre de succession de ces mêmes événements dans l'histoire. Cet exercice, dans Léolo, suscite bien des embarras. D'où un certain désordre ressenti à l'égard de ce récit. Si Léolo ne cherche pas à raconter des événements qui se succèdent, il communique, en revanche, des impressions à partir d'événements singuliers qui datent d'un enfantement rêvé jusqu'à l'enfermement d'un enfant de 12 ans. Cette période de l'enfance se trouve être la ligne thématique principale de l'histoire, c'est-à-dire le récit primaire. À l'intérieur de celui-ci se glissent, semble-t-il, des analepses et des prolepses. À titre d'exemple, je cite une prolepse externe, qui se trouve dans l'épilogue, à la suite de l'internement de Léo. Elle commence ainsi: "Vous la dame, vous l'audacieuse mélancolie qui, d'un cri solitaire, fendez ma chair que vous offrez à l'ennui", et se termine comme suit: "Et j’irai me reposer la tête entre deux mots dans L'Avalée des avalés. [Signé] Léolo." Un autre exemple est celui où Léo et sa mère arrivent à l'hôpital et où la voix off dit: "Mon grand-père, qui n'était pas un homme méchant, avait quand même déjà essayé de me tuer. "Suit l'épisode au cours duquel le grand-père essaie de noyer Léo. Cet épisode semble, à première vue, une analepse quasi complète, un retour en arrière récupérant "l'antécédent » de la visite de Léo et de sa mère à l'hôpital. La voix off revient: "Je me souviens de ne pas avoir eu peur et d'avoir rêvé à la beauté du trésor [...].» D'autres images se mettent en mouvement, évoquant son rêve. Ici, toujours, l'analepse se poursuit, par rapport à Léo marchant dans l'hôpi- 
tal. Tout de suite après apparaît le dompteur de vers qui semble lire le texte que Léo a sans doute écrit après l'événement. Dans ce cas, il s'agit d'une prolepse sur analepse, c'est-à-dire d'une anticipation sur une rétrospection. La voix off parle encore pendant que l'on revoit Léo dans l'eau (analepse) : "Je me souviens de la blancheur de cette lumière que je voyais pour la première fois", puis Léo marche dans les corridors de l'hôpital (récit primaire), retour au grand-père qui se fait assommer par la mère (analepse), Léo à l'hôpital (récit primaire) et, enfin, le grand-père à l'hôpital (récit primaire). Jusque-là, tout porte à croire que Léo et sa mère sont à l'hôpital pour rendre visite au grand-père. Mais quand la voix off reprend, on n'est plus certain de rien... S'agit-il encore d'une analepse quasi complète, puisque: "Quelquefois à l'hôpital, il y avait ma sœur Nanette, ma sœur Rita, mon frère Fernand et puis mon père. Comme si l'hérédité de mon grand-père avait frappé la famille de plein fouet, que la petite cellule de trop s'était déposée dans le cerveau de tout le monde"? Suit alors une courte scène où la psychiatre dit devant toute la famille réunie: "Bonjour, qui parle en premier aujourd'hui? " Ces dernières paroles laissent entendre que la scène s'est répétée plusieurs fois. En effet, Léolo présente très souvent des analepses itératives, "[...] c'est-à-dire portant non sur une seule fraction du temps écoulé, mais sur plusieurs fractions considérées comme semblables et en quelque sorte répétitives [...]" (Genette, 1972, p. 94). Ce type d'analepse renvoie à la question de la fréquence narrative. En voici deux autres exemples. Le premier, pendant que la voix off dit
"Aussi loin que je me souvienne», ce sont les odeurs et la lumière qui ont soudé mes premiers souvenirs. Ma grand-mère avait convaincu mon père que la santé venait en chiant. "Alors tous les vendredis", nous devions subir un traitement choc au laxatif pour nous purifier de toutes les maladies du monde [...]. "Aussi loin que je me souvienne", il y avait un rat dans la baignoire [...]",

plusieurs images montrent notamment Léo à l'âge de deux ans pleurant assis sur une toilette d'enfant, le dompteur de vers lisant en voix in quelques lignes écrites par Léo, Léo plus vieux 
prenant un laxatif, puis se défilant, et retour au Léo de deux ans. Le second exemple:

Mon père était un homme comme tant d'autres. [...] Nanette, ma belle Nanette, je ne t'en veux pas. "Quelquefois", ils étaient au même niveau dans leur délire. Et c'était facile de les visiter «le dimanche matin" dans la grande salle. "Mais d'autres fois", fallait se promener partout pour voir tout le monde. [...] "Y'arrivait même" qu'on doive y passer la journée. "C'était les beaux dimanches de l'hiver".

Les images, quant à elles, s’attardent au père, ensuite à Nanette, etc. Étant donné que ces analepses itératives illustrent que règnent la répétition et l'habitude, peut-être serait-il plus juste, et à l'instar de Genette, de nommer syllepses temporelles («fait de prendre ensemble") ces groupements anachroniques commandés par une parenté thématique (Genette, 1972, p. 121, note 1). Il semble donc que Léolo, au mépris de toute chronologie, mette ensemble des événements liés thématiquement et manifeste, du coup, la capacité d'autonomie temporelle de la narration en regard de l'histoire.

Un dernier exemple de chronologie perturbée est celui de l'enfantement rêvé de Léo. Tout d'abord, la voix off du narrateur:

Ça, c'est chez moi dans le quartier du Mile-End, à Montréal, au Canada. Tout le monde croit que je suis un Canadien français. Parce que moi je rêve, moi je ne le suis pas. Parce que moi je rêve, moi je ne le suis pas. Les gens qui ne croient qu'à leur vérité m’appellent Léo Lauzon. [Apparaît son prétendu père] On dit de lui qu'il est mon père. Mais moi, je sais que je ne suis pas son fils, parce que cet homme est fou et que moi je ne le suis pas. Parce que moi je rêve, moi je ne le suis pas.

Puis, une mention écrite nous transporte en Italie: "Quelque part dans une vallée en Sicile", un homme se masturbe sur un cageot de tomates. La voix off reprend: "Parce qu'il se tenait caché, je n'ai pas vu le visage de mon vrai père.» L'image montre la mère de Léo tombant dans une mare de tomates. Suit la voix off du narrateur: 
Devant l'affolement de ma mère, le docteur n'a pas eu le courage de lui dire qu'elle était enceinte d'une tomate contaminée. "Depuis ce rêve", j’exige qu'on m’appelle Léolo Lozone. Personne n'a le droit de dire que je ne suis pas Italien. L'Italie, c'est trop beau pour n'appartenir qu'aux Italiens.

Il faut bien admettre qu'aucune inférence de contenu ne permet de définir le statut de cette anachronie privée de relation temporelle. S'agissant d'un événement sans date ni âge, on est bien forcé de le considérer comme une achronie. Il est clair qu'à maints endroits, l'ordre chronologique est transgressé par le parti pris de Lauzon de suivre le déroulement d'une mémoire involontaire, de sorte que la reconstitution de l'ordre n'est presque pas possible ni même souhaitable.

Si l'ordre de succession des événements est perturbé, qu'en est-il alors de la vitesse? Pour établir la vitesse d'un récit, on doit confronter la durée de la narration (mesure spatiale) à celle de l'histoire (mesure temporelle). Pour ce faire, j'emprunterai la typologie des formes canoniques de vitesses que sont le sommaire, la pause descriptive, l'ellipse et la scène.

Le sommaire est une narration courte de plusieurs journées, mois ou années d'existence, sans détails d'actions ou de paroles. Ce mouvement, bien que rare dans Léolo, se rencontre à quelques reprises, notamment à la suite de la scène dans laquelle le frère du héros, Fernand, se fait battre par un Anglais. Pendant que le narrateur en voix off dit:

Depuis ce jour, la peur avait donné à mon frère Fernand une raison d'être. Plus jamais Fernand n'aurait peur de personne. Et quand mon frère sera une montagne, moi aussi je n'aurai plus peur et $j$ 'irai dans toutes les ruelles du monde dire aux crottés de cette terre ce que je pense d'eux. Malheur à ceux qui ne pencheront pas la tête sur notre passage. Même les Arabes et les Juifs auront peur de moi tellement je serai haut debout sur les épaules de mon frère.

Les images, quant à elles, montrent Fernand faisant des exercices pour se faire des muscles, puis un Fernand devenu «monsieur muscle". 
La pause descriptive se définit comme un moment où le narrateur abandonne le cours de l'histoire et se charge, pour la seule information du spectateur, de décrire un spectacle que personne ne regarde. Quoiqu'elle soit rarissime dans Léolo, on en trouve un bel exemple dans la scène où toute la famille est réunie autour de la table de la cuisine. La voix off décrit le père: "Mon père était un homme comme tant d'autres. Un chien qui mordait dans sa chienne de vie. C'était un petit, baquet, aux pommettes rouges [...]. " L'image, mobile au départ, s'immobilise sur le père assis à la table; s'ajoutent d'autres images concernant le père, puis l'image revient à la table où personne ne semble intéressé à rien... Au total, ni le sommaire ni la pause descriptive ne sont, dans Léolo, frappants ou significatifs.

On l'aura compris, Léolo est truffé d'ellipses, c'est-à-dire de temps d'histoire élidés et de scènes qui instituent une sorte d'égalité conventionnelle entre le temps de la narration et le temps de l'histoire. Deux exemples permettront d'illustrer cet aspect du film. Le premier est une scène du prologue, qui est immédiatement suivic d'une ellipse: celle où la mère de Léo tombe dans le cageot de tomates. Le second est une scène assez longue au cours de laquelle on suit Léo pas à pas. Elle arrive après le titre et se termine également par une ellipse. Léo ouvre la porte extérieure et fait pipi sur le balcon, sa mère lui crie de l'intérieur de l'appartement d'aller dormir, il hurle à son tour: "Léolo, Léolo Lozone, je m’appelle Léolo", il rentre, s’installe pour lire à la lumière du réfrigérateur L'Avalée des avalés. Quand il cesse de lire et s'arrête à la page de titre où est écrit à la main "parce que moi je rêve, moi je ne le suis pas", la voix off répétera au plus quatre fois cette phrase qui mettra fin à la scène. Il va sans dire que la longueur des scènes de Léolo n'est pas comparable à celle des scènes de la Recherche de Proust. Qu'on se souvienne du souper chez Guermantes, scène exemplaire, qui couvre au-delà de cent pages du "Côté de Guermantes» (1983, p. 416-547). Cependant, grâce à la voix off du narrateur, on sait que la scène de la lecture est initiatique: "Au début", j’en lisais des passages soulignés sans trop comprendre. Je me souviens d'avoir voulu abandonner [...].» Comme dans la Recherche, plusieurs scènes de Léolo sont typiques ou exem- 
plaires. Une scène en vaut mille autres, par analogie. S'explique ici encore, donc, le choix de Lauzon de conduire le récit filmique à coups d'associations libres.

Si le mouvement du texte lauzonnien est surtout cadencé au rythme de la scène et de l'ellipse, comment peut-on avoir l'impression d'une vue d'ensemble sur l'enfance de Léo? Pour répondre à cette question, il faut faire appel à la notion de capacité de répétition de la narration, c'est-à-dire à la fréquence narrative ou, mieux encore, aux relations de répétition entre la narration et l'histoire.

Comme pour la Recherche, Léolo est une narration principalement itérative, c'est-à-dire qu'en une seule fois, le narrateur raconte ce qui s'est passé plusieurs fois. Léolo raconte, en effet, "[...] à l'imparfait de répétition, non ce qui s'est passé, mais ce qui se passait" (Genette, 1972, p. 149) dans l'enfance de Léo: "Alors "tous les vendredis" nous " devions " subir un traitement choc au laxatif pour nous purifier de toutes les maladies du monde. La marde "était" devenue une obsession dans la famille." Bien qu'il ne soit pas "[...] facile d'exprimer qu'une action montrée à l'écran vaut pour plusieurs actions similaires" (Gaudreault et Jost, p. 123), la voix off du narrateur pallie cette difficulté:

"Il m’a fallu longtemps» pour comprendre qu'il était la réincarnation de Don Quichotte et qu'il avait décidé de se battre contre l'ilotisme et de me protéger du gouffre de ma famille. [...] "Rarement», il nous arrivait d'être tous ensernble à la maison, où on avait l'air d'être une vraie famille. [...] C'était Bianca, qui, "depuis longtemps", chantait pour moi dans le fond du placard. Il suffisait de prendre le temps d'écrire. [...] $\mathrm{Et}$, «comme toujours», je me regardais jouer à la vie.

Quant à la narration répétitive, il s'agit de récurrences de la narration qui ne se retrouvent pas dans les événements. Ici se place le leitmotiv du film: «Parce que moi je rêve, moi je ne le suis pas. " Dans l'image, la première fois qu'il est donné à voir, c'est lorsque Léo lit le livre de Réjean Ducharme. La première fois qu'il est donné à entendre, c'est durant le prologue. Il est d'ailleurs énoncé au moins deux fois à cette occasion. Il sera 
répété six autres fois et remplacé par une autre répétition : "Je ne rêve plus. Je ne rêve plus."

Enfin, la narration singulative, qui se définit par l'égalité du nombre de ses occurrences dans la narration ainsi que dans l'histoire, se rencontre lorsque le narrateur dit: "[...] puis il y eut "un soir" où il y avait une dinde que ma mère avait gagnée au cinéma. Elle était grosse et sale. Ce qui lui restait de plumes était tout humide et sentait mauvais." Dans Léolo, ni la narration singulative ni la narration répétitive ne portent à conséquence. Ce qui fait l'originalité du film de Lauzon, c'est l'utilisation abondante de l'itératif.

Grâce au film de Lauzon, il a été possible d'exhumer un narrateur qui avait été enterré vivant. Grâce également à cette narratologie comparée, sans laquelle il aurait été malaisé d'identifier - tant on avait bien appris la leçon des narratologues - ou même de nommer — sans que soient créés d'autres néologismes - ce phénomène que je prétends aussi répandu dans le récit filmique que dans le récit littéraire. En tant qu'instance narrative, Léo ou Léolo représente un type, à savoir le narrateur extradiégétique-homodiégétique, qui manquait tant à la théorie filmique. Léo est, en effet, l'unique narrateur de ce Léolo "égocentrique». Il est la seule instance à ouvrir la bouche, à dire "je", pour raconter son histoire, ce que l'on désigne, en termes narratologiques, par narrateur extradiégétique-homodiégétique, voire autodiégétique. Ce dernier est incarné par un Léo plus âgé que celui aperçu à l'écran, puisque la narration est ultérieure. De plus, à quelques exceptions près, il est le foyer de toutes les perceptions du film. Pour clore sur le parallèle entre Léolo et la Recherche de Proust, je dirais avec Genette que "[...] la syllepse itérative n'est pas seulement un fait de fréquence" (1972, p. 18), puisqu'elle touche aussi bien l'ordre du film que sa durée. C'est elle qui conditionne les scènes et les ellipses; c'est elle qui pervertit l'ordre; c'est elle, aussi, qui oblige une telle présence du narrateur. C'est elle, enfin, qui permet aux impressions (à la mémoire involontaire) de se dire sans perte de sens. Dans Léolo, c'est la narration itérative qui exacerbe la présence du narrateur en voix off. Et c'est cette présence accentuée qui conduit au 
rythme inhabituel du film de Lauzon. C'est d'ailleurs à ce rythme syncopé, qui provoque une rupture chronologique (chaos des images), que s'en sont pris certains critiques qui y voyaient une influence directe du monde de la pub et du vidéo. Désordre, oui, mais désordre "organisé", puisqu'il suit l'ordre "anarchique» des associations de la mémoire involontaire qui tiennent du subconscient plus que de la séquence linéaire du rationnel. Choisir la ligne de l'imaginaire et réussir à évoquer une enfance tient de l'art cinématographique, n'en déplaise aux critiques.

\section{Chercheure autonome}

\section{NOTES}

1 Ce segment du titre entre guillemets est emprunté à l'article de Michel Rebichon, "Un film cru. Une enfance nue" (Studio, n 65, 1992, p. 22); quant au présent article, il est en partie une refonte d'une conférence donnée en 1993 au colloque de l'AQEC.

2 "Tel qu'il est, ce Léolo brillant, inégal, égocentrique, est un des moments forts de notre cinéma et une ouuvre fascinante à voir et à revoir " (Odile Tremblay, "Des fragments de rêves fascinants", Le Devoir, 6 juin 1992, p. C-3). "Tout ce qui brille n'est pas or. Et ce n'est pas parce que Léolo a été sélectionné pour la compétition officielle du dernier festival de Cannes, qu'il faut, comme avec les vessies et les lanternes, le prendre pour un chef-d'œuvre [...]. Jean-Claude Lauzon, qui travaille dans la publicité [...], se montre habile dans l'illusion, et il faut voir son film deux fois plutôt qu'une pour y déceler cette tendance d'un cinéma de l'illustration [...], un cinéma culturel, noble, digne de tous les publics, impeccable; un cinéma de qualité, somme toute, qui trafique un nouvel académisme dont l'héritage tient plus de la pub et du vidéo-clip que du cinéma " (André Roy, "Léo pour Léolo ou du pareil au même", Spirale, n"117, 1992, p. 16).

3 Du moins en ce qui concerne Voix et Mode: Bal l'a répété à l'envi.

4 Je me suis expliquée, à l'occasion d'une conférence, sur le fait que les termes "absent" et "étranger" ne me paraissaient pas parfaitement synonymes. Voir "Les métalepses narratives de Kamouraska ou comment l'auteure s'immisce dans son propre texte (jusqu'à y devenir l'un des protagonistes?)" (Anne Hébert, parcours d'une auvre, Montréal: l'Hexagone 1997, p. 265-278).

5 Comme le rappelle Metz dans la préface de la traduction française du livre de Casetti, ce dernier est le premier au cinéma "[...] à avoir proposé des éléments de formalisation pour l'ensemble de l'appareil énonciatif du film" (D'un regard l'autre. Le film et son spectateur, Lyon: Presses universitaires de Lyon, 1990, p. 8).

6 On le voit, en théorie narratologique filmique, on use des termes narration et énonciation, ce qui a parfois pour effer de créer une certaine confusion; pour Jost, à titre d'exemple, l'énonciation cinématographique renvoie au style du supposéréalisateur en tant qu'il parle cinéma et l'énonciation narrative (ou narration) à celui qui raconte, c'est-à-dire au narrateur implicite. Sur cette question, voir Glenda 
Wagner et François Jost, "François Jost: Un monde à notre image. Énonciation, cinéma, télévision" (Études littéraires, vol. 26, n"2,1993, p. 114) et aussi dans André Gaudreault et François Jost, Le Récit cinématographique. Cinéma et Récit II (Paris: Nathan, 1990): "[...] ce moment où le spectateur s'échappant de l'effet-fiction aurait la conviction d'être en présence du langage cinématographique comme tel " (p. 44).

7 Tous les exemples de Gaudreault pour illustrer son narrateur délégué sont des instances effectivement intradiégétiques-homodiégétiques: Romano des Yeux noirs, Walter Neff de Double Indemnity, Von Stratten de Confidential Report (Du littéraire au filmique. Système du récit, Paris / Québec: Méridiens Klincksieck / Presses de l'Université Laval, 1988, p. 158). Romano s'adresse à un narrataire de l'histoire, Pavel; Neff confesse son crime, par le truchement d'un magnétophone, à son collègue de travail; Van Stratten expose à Zouk leur infortune.

8 Selon ses propres termes, le film contient toujours « [...] un grand imagier polyphonique [...] qui ne cède à son délégué qu'un seul canal, celui de la parole, et qui reste l'origine illocutoire, sans délégation aucune, de ces images, de ces bruits, de ces musiques et, éventuellement, de ces mentions écrites qui enserrent ce discours narratif de niveau second que nous tiennent tous les Romano filmiques du monde" (André Gaudreault, Du littéraire au filmique. Système du récit, Paris / Québec: Méridiens Klincksieck / Presses de l'Université Laval, 1988, p. 178).

9 "[Le] locuteur premier, le narrateur implicite, c'est celui qui "parle" cinéma au moyen des images et des sons; le narrateur explicite lui ne raconte qu'avec des mots" (André Gaudreault et François Jost, Le Récit cinématographique. Cinéma et Récit II, Paris: Nathan, 1990, p. 48); "[II] est une seule façon non métaphorique de raconter à la "première personne". Et c'est cette façon qui consiste à utiliser, précisément, et "là où c'est possible", la "première personne" [...]. Or s'il est possible pour l'instance apparemment première du récit scriptural et pour celle qui est fondamentalement responsable du récit oral de se désigner, soi, par l'utilisation de ce déictique par excellence, la chose est ontologiquement impossible pour l'instance responsable, fondamentalement, du récit filmique, instance qui ne peut dire " je" [...] car elle ne parle pas français [...]. Elle "parle", comme le dit Jost, "cinéma". Et, tout au plus peut-elle, cette instance, "faire dire" "je" par une autre instance qui, d'une part, lui sera "subordonnée" "et, d'autre part, devra nécessairement, pour ce faire "sincarner", "s'actorialiser" "(André Gaudreault, Du littéraire au filmique. Système du récit, Paris I Québec: Méridiens Klincksieck / Presses de l'Université Laval, 1988, p. 177).

10 "Si l'on envisage (définition large) toute espèce de "représentation" d'une histoire, il y a évidemment récit théâtral, récit filmique, récit par bandes dessinées, etc. Personnellement, je suis plutôt, et de plus en plus, pour une définition étroite de récit: haplè diègèsis, exposé des faits par un narrateur qui [les] signifie par voie verbale (orale ou écrite), et en ce sens il n'y a pas pour moi de récit théâtral ou filmique. Le théâtre "ne raconte pas", il "reconstitue" une histoire sur scène, et le cinéma "montre" sur l'écran une histoire "reconstituée" (en fait, bien sûr, constituée) sur le plateau" (Genette, cité par André Gaudreault, Du littéraire au filmique. Système du récit, Paris / Québec: Méridiens Klincksieck / Presses de l'Université Laval, 1988, p. 29, note 8). À ce sujet, je pense comme Mathieu-Colas que si Genette «[...] a pour lui l'autorité de la tradition" " Frontières de la narratologie", Poétique, n" 65, 1986, p. 93) - à savoir que si le récit désigne, par priorité, "une relation orale" avant de s'appliquer, par extension, à l'écriture, les dictionnaires contemporains n'en souscrivent pas moins à ce qu'il ne peut être donné que de vive voix ou par écrit -, que cette tradition donc " [...] ne peut pas suffire à rendre compte des systèmes de communication développés dans le cadre de la culture contemporaine" (p. 94). Ainsi, il n'y a pas d'autre solution que d'admettre conjointement un sens restreint et un sens large, et qu'il faudra se satisfaire de la dualité - si incommode, si gênante soit-elle. 
11 Metz affirme, pour sa part, que ceux qui estiment qu'énonciation au cinéma signifie quelque chose ne doivent pas prendre à la légère cet argument de Genette, qui est en fait très fort. "Il nous oblige à une importante reconversion: à concevoir un appareil énonciatif qui ne soit pas essentiellement déictique (et donc anthropomorphique), pas personnel (comme les pronoms que l'on appelle ainsi), et qui n'imite pas de trop près tel ou tel dispositif linguistique, car l'inspiration linguistique réussit mieux de loin" (L'Énonciation impersonnelle ou le site du film, Paris: Méridiens Klincksieck, 1991, p. 19).

12 Je citerai tour à tour trois narratologues. Alors que Genette précise, il est vrai, qu'il s'agit "essentiellement d'une méthode" et évoque la scientificité (pour rire?), Bal et Gaudreault désignent expressément la narratologie comme une science. D’ailleurs, semble-t-il, cette acception, même tacite, a remporté l'adhésion de nombreuses personnes qui se sont penchées sur la narratologie. "Ce que je propose ici est essenticllement une méthode d'analyse" (Figures III, Paris: Seuil, 1972, p. 68). "Mais cautionner en scientificité un vertige, voire un strabisme mérhodologicue tha théoric littéraire et la critiquel, ne va pas sans imposture. Je plaiderai donc autrement la même cause: peut-être la véritable relation entre l'aridité "théorique" et la minuric critique est-elle ici [faite] d'alternance et de distraction réciproque" (69); " $|\mathrm{la}|$ narratologie est la science qui cherche à formuler la théorie des textes narratifs dans leur narrativité" (Mieke Bal, Narratologie. Les Instances du récit, Paris: Klincksieck, 1977, p. 4); "[d'où] la nécessité de "bouter" l'Auteur hors de la Narratologie, qui est [...] une science s'occupant du récit et des récitants, pas de ceux qui les créent" (André Gaudreault, Du littéraire au filmique. Système du récit, Paris / Québec: Méridiens Klincksieck / Presses de l'Université Laval, 1988, p. 154).

13. Voir le texte intégral de Genette conforme à ce dernier, Fiction et Diction (Paris: Seuil, 1991). Quant à la notion d'auteur, Genette s'exprime comme suit : "Je ne suis pas sûr de rester dans les limites du champ proprement narratologique en évoquant, au titre des questions de voix ("Qui parle?"), le sujet toujours épineux des rapports entre le narrateur et l'auteur" (p. 79); et dans L'CEuvre de l'art: "[...] la promotion, parfois naïve, dans nos années soixante-dix, de la notion de "rexte" aux dépens de celle d" œuvre" [...]. La destitution "formaliste" de l'intention auctoriale, poussec symboliquement jusqu'au meurtre, et la valorisation "structuraliste" de l'autonomie du texte [...]" (Paris: Seuil, 1994, p. 223).

14. Je propose, pour les distinguer, que la sous-catégorie du «temps de la narration» de Genette au chapitre de la Voix devienne le "moment de la narration" et que le "temps du récit" de la catégorie du Temps soit remplacé par le "temps de la narration".

\section{OUVRAGES CITÉS}

Bal, Mieke. Narratologie. Les Instances du récit. Paris: Klincksieck, 1977.

Barthes, Roland. "La mort de l'auteur", Le Bruissement de La langue. Essais critiques IV. Paris: Seuil (1984), p. 63-69.

Benveniste, Émile. Problèmes de linguistique générale II. Paris: Gallimard, 1974.

Bordwell, David. Narration in the Fiction Film. Madison: University of Wisconsin Press, 1985.

Caserti, Franscesco. D'un regard l'autre. Le film et son spectateur. Lyon: Presses Universitaires de Lyon, 1990.

Cohn, Dorrit. "Signposts of Fictionality: A Narratological Perspective", Poetics Today, vol. 11, n" 4 (1590), p. 775-804.

Pour poser les jalons d'une narratologie comparée: Léolo à la "recherche d'un temps..." 201 
Gaudreault, André. Du littéraire au filmique. Système du récit. Paris / Québec: Méridiens Klincksieck / Presses de l'Université Laval, 1988.

Gaudreault, André et François Jost. Le Récit cinématographique. Cinéma et Récit II. Paris: Nathan, 1990.

Genette, Gérard. L'CEuvre de l'art. Immanence et transcendance. Paris: Seuil, 1994.

Genette, Gérard. Fiction et Diction. Paris: Seuil, 1991.

Genette, Gérard. Nouveau Discours du récit. Paris: Seuil, 1983.

Genette, Gérard. Palimpsestes. La Littérature au second degré. Paris: Seuil, 1982.

Genette, Gérard. Figures III. Paris: Seuil, 1972.

Hamburger, Käte. Logique des genres littéraires. Paris: Seuil, 1986.

Jost, François. "La sémiologie du cinéma et ses modèles ". Iris, n" 10 (1990), p. 133141.

Mathieu-Colas, Michel. "Frontières de la narratologie". Poétique, n" 65 (1986), p. $91-110$.

Metz, Christian. "L'énonciation impersonnelle ou le site du film". Vertigo, n" 1 , (1987), p. 15-34.

Metz, Christian. L'Énonciation impersonnelle ou le site du film. Paris: Méridiens Klincksieck, 1991.

Proust, Marcel. À la recherche du temps perdu, tome II. Paris : La Pléiade, 1983.

Proust, Marcel. Contre Sainte-Beuve. Paris: Gallimard, 1954.

Rebichon, Michel. "Un film cru. Une enfance nue». Studio, n" 65 (1992), p. 22.

Reboul, Olivier. La Rhétorique. Paris: Presses universitaires de France, 1990.

Roy, André. «Léo pour Léolo ou du pareil au même». Spirale, n” 117 (1992), p. 16.

Saussure, Ferdinand de. Cours de linguistique générale. Paris: Payot, 1972.

Steiner, George. Réelles présences. Les arts du sens. Paris: Gallimard, 1990.

Tremblay, Odile. "Des fragments de rêves fascinants". Le Devoir, 6 juin 1992, p. C-3.

Wagner, Glenda et François Jost. "François Jost: Un monde à notre image. Énonciation, cinéma, télévision». Études littéraires, vol. 26, n" 2 (1993), p. 105-109.

Wagner, Glenda. "Les métalepses narratives de Kamouraska ou comment l'auteure s'immisce dans son propre texte (jusqu'à y devenir l'un des protagonistes?)", Anne Hébert, parcours d'une ouvre. Montréal : l'Hexagone, 1997, p. 265-278. 